\title{
Bandgap behavior and singularity of the domain-induced light scattering through the pressure-induced ferroelectric transition in relaxor ferroelectric $\mathrm{A}_{x} \mathrm{Ba}_{1-x} \mathrm{Nb}_{2} \mathrm{O}_{6}(\mathrm{~A}$ : \\ $\mathrm{Sr}, \mathrm{Ca})$ \\ J. Ruiz-Fuertes, O. Gomis, A. Segura, M. Bettinelli, M. Burianek, and M. Mühlberg
}

Citation: Appl. Phys. Lett. 112, 042901 (2018);

View online: https://doi.org/10.1063/1.5012111

View Table of Contents: http://aip.scitation.org/toc/apl/112/4

Published by the American Institute of Physics

\section{Articles you may be interested in}

lonic transport and dielectric properties in $\mathrm{NaNbO}_{3}$ under high pressure

Applied Physics Letters 111, 152903 (2017); 10.1063/1.4999206

Origin of the dielectric abnormities and tunable dielectric properties in doped KTN single crystals Applied Physics Letters 111, 242902 (2017); 10.1063/1.5005035

Polymer/metal multi-layers structured composites: A route to high dielectric constant and suppressed dielectric loss

Applied Physics Letters 112, 022901 (2018); 10.1063/1.5009795

Influence of impurities on the high temperature conductivity of $\mathrm{SrTiO}_{3}$

Applied Physics Letters 112, 022902 (2018); 10.1063/1.5000363

Intrinsic terahertz photoluminescence from semiconductors

Applied Physics Letters 112, 041101 (2018); 10.1063/1.5012836

Effect of finger geometries on strain response of interdigitated capacitor based soft strain sensors

Applied Physics Letters 112, 044101 (2018); 10.1063/1.4998440

\section{Scilight}

Sharp, quick summaries illuminating the latest physics research

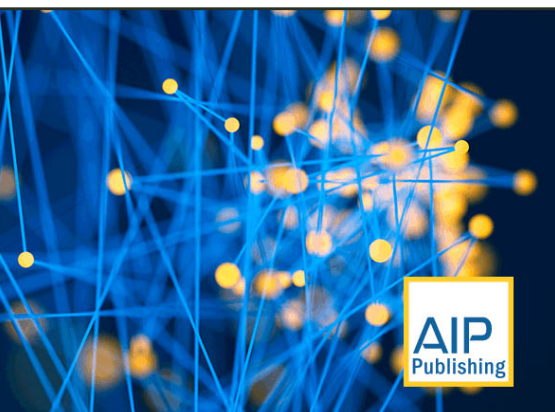




\title{
Bandgap behavior and singularity of the domain-induced light scattering through the pressure-induced ferroelectric transition in relaxor ferroelectric $A_{x} \mathrm{Ba}_{1-x} \mathrm{Nb}_{2} \mathrm{O}_{6}(A: \mathrm{Sr}, \mathrm{Ca})$
}

\author{
J. Ruiz-Fuertes, ${ }^{1,2, a)}$ O. Gomis, ${ }^{3}$ A. Segura, ${ }^{2}$ M. Bettinelli, ${ }^{4}$ M. Burianek, ${ }^{5}$ and M. Mühlberg ${ }^{5}$ \\ ${ }^{1}$ DCITIMAC, MALTA Consolider Team, Universidad de Cantabria, 39005 Santander, Spain \\ ${ }^{2}$ Departament de Física Aplicada-ICMUV, MALTA Consolider Team, Universitat de València, \\ 46100 Burjassot, Spain \\ ${ }^{3}$ Centro de Tecnologías Físicas, MALTA Consolider Team, Universitat Politècnica de València, \\ 46022 València, Spain \\ ${ }^{4}$ Laboratorio Materiali Luminescenti, DB, Università di Verona, and INSTM, UdR Verona, 37134 Verona, \\ Italy \\ ${ }^{5}$ Institut für Kristallographie, Universität zu Köln, 50937 Köln, Germany
}

(Received 6 November 2017; accepted 9 January 2018; published online 23 January 2018)

\begin{abstract}
In this letter, we have investigated the electronic structure of $A_{x} \mathrm{Ba}_{1-x} \mathrm{Nb}_{2} \mathrm{O}_{6}$ relaxor ferroelectrics on the basis of optical absorption spectroscopy in unpoled single crystals with $A=\mathrm{Sr}$ and $\mathrm{Ca}$ under high pressure. The direct character of the fundamental transition could be established by fitting Urbach's rule to the photon energy dependence of the absorption edge yielding bandgaps of 3.44(1) $\mathrm{eV}$ and 3.57(1) $\mathrm{eV}$ for $A=\mathrm{Sr}$ and $\mathrm{Ca}$, respectively. The light scattering by ferroelectric domains in the pre-edge spectral range has been studied as a function of composition and pressure. After confirming with x-ray diffraction the occurrence of the previously observed ferroelectric to paraelelectric phase transition at $4 \mathrm{GPa}$, the light scattering produced by micro- and nanoferroelectric domains at $3.3 \mathrm{eV}$ in $\mathrm{Ca}_{0.28} \mathrm{Ba}_{0.72} \mathrm{Nb}_{2} \mathrm{O}_{6}$ has been probed. The direct bandgap remains virtually constant under compression with a drop of only $0.01 \mathrm{eV}$ around the phase transition. Interestingly, we have also found that light scattering by the polar nanoregions in the paraelectric phase is comparable to the dispersion due to ferroelectric microdomains in the ferroelectric state. Finally, we have obtained that the bulk modulus of the ferroelectric phase of $\mathrm{Ca}_{0.28} \mathrm{Ba}_{0.72} \mathrm{Nb}_{2} \mathrm{O}_{6}$ is $B_{0}=222$ (9) GPa. Published by AIP Publishing. https://doi.org/10.1063/1.5012111
\end{abstract}

The tungsten-bronze family of relaxor ferroelectrics $\left(A_{x} \mathrm{Ba}_{1-x} \mathrm{Nb}_{2} \mathrm{O}_{6}, A \mathrm{BN} x, A=\mathrm{Sr}\right.$ and $\left.\mathrm{Ca}\right)$ is being intensively studied for frequency conversion processes, data storage, optical computing, or as non-linear prisms. ${ }^{1-4}$ Despite its importance in the development of applications with these technologically important materials, fundamental parameters of their electronic structure like the bandgap remain unknown. Optical absorption measurements on $A B N x$ samples with thicknesses ranging from $0.3 \mathrm{~mm}(\mathrm{SBN} 61)^{5,6}$ to $2.51 \mathrm{~mm}(\mathrm{CBN} 28)^{7}$ suggest a blueshift of the absorption edge of CBN28 with respect to SBN61 and a continuous behavior across the ferroelectric to paraelectric phase transition that occurs at $T_{N}=539$ and $T_{N}=352 \mathrm{~K}$ in CBN28 and SBN61, respectively. However, the accurate determination of the absorption coefficient, $\alpha,{ }^{8}$ to obtain the bandgap demands sample thicknesses, $d$, ensuring $\alpha d \sim 1$, not accomplished by previous studies ${ }^{5-7}$ which only report the temperature dependence of the energy at which the absorption coefficient $\alpha$ gets a value of $100 \mathrm{~cm}^{-1}$.

Otherwise, ABN crystallizes in the partially filled tetragonal tungsten-bronze-type structure in which only five of the six A1 and A2 voids are occupied with strontium/calcium and barium cations in a modulated fashion described in the P4bm(aa1/2; -aa1/2) superspace group. ${ }^{9-11}$ Such structural modulated disorder prevents any reliable calculation of the

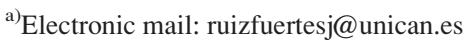

electronic structure and reinforces the importance of an accurate experimental determination of the bandgap and character of the fundamental transition of these compounds.

In this letter, we report on non-polarized optical absorption measurements on unpoled tungsten-bronze-type relaxor ferroelectric single crystals with different compositions (SBN40, SBN50, SBN66, and CBN28). Also, we confirm with $\mathrm{X}$-ray diffraction (XRD) the pressure-induced structural phase transition of CBN28 from the ferroelectric to the paraelectric phase ${ }^{12}$ and explore the pressure effect on the shape of its absorption edge at ambient temperature.

The SBN $x$ single crystals $(x=0.4,0.5$, and 0.66$)$ studied here were grown from fluxes consisting of mixtures of barium and strontium tetraborates in the temperature range of 900-1300 ${ }^{\circ} \mathrm{C} .{ }^{13,14}$ The CBN28 single crystals were grown by the Czochralski method. ${ }^{15}$ For optical absorption in the UVVIS range, we employed non-oriented samples with the thickness between 10 and $20 \mu \mathrm{m}$ and a confocal optical setup consisting of a deuterium lamp, fused silica lenses, two Cassegrain objectives, and an UV-VIS spectrometer. For the optical absorption experiments under pressure, the same setup was used with a beam spot of $25 \mu \mathrm{m}$. The sample was placed together with a ruby chip for pressure determination ${ }^{16}$ into the $250 \mu \mathrm{m}$ diameter drilled hole of $50 \mu \mathrm{m}$ thick stainless steel gasket inserted between two IIA diamonds of a membrane-type diamond anvil cell (DAC). A mixture of methanol-ethanol (4:1) was used as pressure transmitting 
medium. The absorption spectra were derived from transmission measurements performed on $\sim 10-15 \mu$ m-thick single crystals exfoliated from the $\sim 2 \times 2 \times 1 \mathrm{~mm}^{3}$ crystals obtained in the growth. The powder XRD experiment was carried out with a powder pellet of ground CBN28 inserted together with a ruby chip and a mixture of methanol-ethanol (4:1) inside a membrane-type DAC with a stainless steel gasket indented to $30 \mu \mathrm{m}$ and a hole of $150 \mu \mathrm{m}$ at the ALBACELLS synchrotron ${ }^{17}$ using a wavelength of $\lambda=0.4246 \AA$ and a sample to detector distance of $209.96 \mathrm{~mm}$.

The absorption edges of the four tungsten-bronze-type compounds studied at ambient pressure are shown in Fig. 1. It should be emphasized that the explored range absorption coefficient values are 45 -fold higher than previous measurements limited to $\alpha_{\max }=120 \mathrm{~cm}^{-1}$, thus providing more information in the high-energy range of the spectrum. This is basic to be able to both quantify the bandgap and determine the character of the fundamental absorption. The high values of alpha in the four compounds and the steepness of the absorption edge indicate a direct character of the fundamental absorption. Such character is confirmed by the excellent fits shown in Fig. 1 with an Urbach's tail ${ }^{18}$ according to $\alpha=A_{0} \cdot \exp \left[\left(E-E_{g}\right) / E_{U}\right]$. Given that only the Urbach's energy, $E_{U}$, can be independently determined (from the slope of the semilogarithmic plot) and the absorption intensity depends on both $A_{0}$ and the bandgap $E_{g}$, we fixed $A_{0}$ to $500 \mathrm{~cm}^{-1}$ which is the absorption level found in other direct bandgap compounds showing similar maximum values of $\alpha$ for similar sample thicknesses. ${ }^{19}$ This strategy showed that the value of $E_{U}$, related to the
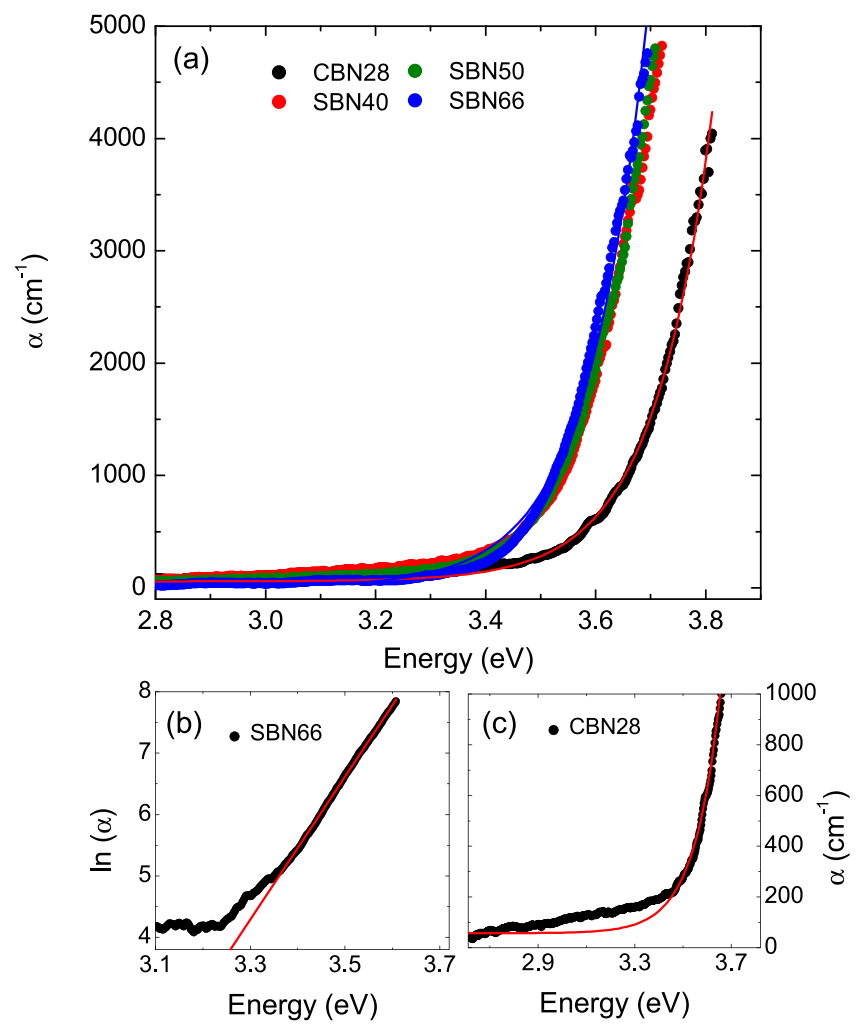

FIG. 1. (a) Absorption coefficient of SBN40, SBN50, SBN66, and CBN28 determined in $10 \mu \mathrm{m}$ thick crystals. (b) Linear fit of the $\ln (\alpha)$ showing the agreement between Urbach' law and the experimental data points of the absorption spectrum of SBN66. (c) Zoom of part of the absorption spectra of CBN28 that deviates from Urbach's behavior. The points are the experimental data and the continuous lines fit with Urbach's law. steepness of the absorption edge, is virtually the same for the three compounds, $E_{U}=0.114(3) \mathrm{eV}$, and provided the bandgaps $E_{g}$ summarized in Table I.

With the increase of the Sr content in SBNx, the absorption edge seems to slightly blueshift but basically remains constant. However, the absorption edge of CBN28 clearly appears shifted to higher energies with respect to SBN $x$, indicating a widening of the bandgap when $\mathrm{Sr}$ is substituted by $\mathrm{Ca}$ (Table I). In the tungsten-bronze-type structure, both $\mathrm{Nb}^{5+}$ ions are octahedrally coordinated in a distorted symmetry where $\mathrm{Nb}^{5+}$ is shifted along the $c$ axis breaking the center of inversion in the structure. While no detailed orbital decomposition of the electronic density of states currently exists for the electronic structure ${ }^{20}$ of $\mathrm{Nb}_{2} \mathrm{O}_{5}$, we can consider the orbital projection of the density of states ${ }^{21}$ of $\mathrm{V}_{2} \mathrm{O}_{5}$ where $\mathrm{V}^{5+}$ is also octahedrally coordinated for comparison with our compounds as a first approximation. Similar to $\mathrm{V}_{2} \mathrm{O}_{5}$, we can expect that the ground state of $\mathrm{ABN} x$ is formed by a valence band top mainly contributed by completely filled O $2 p$ orbitals and a conduction band bottom contributed by unoccupied $\mathrm{Nb} 4 d$ orbitals $\left(\mathrm{Nb}^{5+}, 4 d^{0}\right)$. Moreover, due to the octahedral surrounding of the niobium ions, we can expect a crystal-field splitting of the $\mathrm{Nb} 4 d$ orbitals into $t_{2 g}$ states at the lower edge of the conduction band and $e_{g}$ bands located at higher energies. In this picture, consistent with the pressure insensitivity of the bandgap discussed below in this paper, the $\mathrm{Ca}, \mathrm{Sr}$, and $\mathrm{Ba}$ outer-shell $s$ orbitals would not contribute much at the top (bottom) of the valence (conduction) band which is a good assumption if we compare with other oxides containing an alkaline earth metal and a $d^{0}$ transition metal. ${ }^{22}$ Thus, the difference in the bandgap between SBN $x$ and CBN28 could most probably be explained in the frame of $\mathrm{Nb}-\mathrm{O}$ bond distance differences and distortion in the $\mathrm{NbO}_{6}^{8-}$ polyhedra. In general, the $\mathrm{O} \pi$ and $\sigma$ orbitals split faster with a shortening of the $\mathrm{Nb}-\mathrm{O}$ bond distances than the $\mathrm{Nb} t_{2 g}$ and $e_{g}$ states $^{23,24}$ as a result of a crystal field increase. This implies that a reduction of the $\mathrm{Nb}-\mathrm{O}$ bond length would shift the $\mathrm{O} 2 p$ orbitals up in the valence band faster than the $\mathrm{Nb} 4 d$ would split at the bottom of the conduction band, thus reducing the bandgap. However, previous structural works show that the variation of the mean $\mathrm{Nb}-\mathrm{O}$ bond length in $\mathrm{SBN} x$ reduces from 2.14 to $1.8 \AA$ with the $\mathrm{Sr}$ concentration, ${ }^{11}$ and it is $\sim 1.97 \AA$ in CBN28. ${ }^{10}$ Therefore, the reduction of the mean Nb-O bond length cannot account alone for the increase observed in the bandgap of CBN28 with respect to SBN $x$ and the distortion of the polyhedra needs to be considered. If we compare the off-centering along $c$ in both $\mathrm{NbO}_{6}^{8-}$ octahedra, we find that the up-shift of the $\mathrm{Nb}^{5+}$ ions in CBN28 with $0.2 \AA$ doubles ${ }^{10}$

TABLE I. Energy of the fundamental bandgap $E_{g}$ as obtained from the fit with an Urbach's tail in the studied relaxor ferroelectrics and the value of the absorption coefficient $\alpha$ at the pre-edge $(3.3 \mathrm{eV})$ as a result of photon dispersion with ferroelectric microdomains present in our unpoled single crystals.

\begin{tabular}{lcccc}
\hline \hline & SBN40 & SBN50 & SBN66 & CBN28 \\
\hline$E_{g}(\mathrm{eV})$ & $3.44(1)$ & $3.44(1)$ & $3.44(1)$ & $3.57(1)$ \\
$\alpha\left(\mathrm{cm}^{-1}\right)$ & 224 & 179 & 117 & 155 \\
\hline \hline
\end{tabular}


the value of $\mathrm{SBN} 20^{9}$ of $0.106 \AA$. This characteristic, responsible for conferring CBN28 the highest spontaneous polarization, ${ }^{25} 35.3 \mu \mathrm{C} / \mathrm{cm}^{2}$, and the highest Curie temperature within the tungsten-bronze-type relaxor ferroelectrics, should most likely affect the bottom of the conduction band probably reducing the total crystal field and therefore the $e_{g}$ and $t_{2 g}$ splitting of the empty $\mathrm{Nb} 4 d$ orbitals. This hypothesis, which is in part supported by our high-pressure study presented below, calls for electronic band structure calculations which, unfortunately, cannot be reliably performed in these modulated and disordered compounds.

In Figs. 1(a) and 1(b), we have shown the good agreement between the experimental and simulated absorption spectra using an Urbach's tail. However, in Fig. 1(c), we can appreciate that the low-energy part of the spectrum cannot be successfully accounted by the fit. In fact, in Table I, we show that the value of $\alpha$ at the pre-edge $(3.3 \mathrm{eV})$ for the four compounds changes with the $\mathrm{Sr}$ content in $\mathrm{SBN} x$. The absorption edge of most direct bandgap compounds usually presents an exponential pre-edge tail superposed to the main edge. Figure 1(c) shows how the experimental data deviate from the exponential decrease in the pre-edge region. It is known ${ }^{26}$ that in unpoled ferroelectrics, the existence of ferroelectric microdomain walls produces a strong light scattering just below the pre-edge as a result of refractive index changes between the domains originated from the large index dispersion near the absorption edge resulting from the intense local electric field. Light scattering in transmission experiments as ours is detected through the appearance of an absorption tail. A similar effect, observed in $\mathrm{SBN}_{6} 1^{27,28}$ and other $^{29}$ ferroelectrics as $\mathrm{Pb}\left[\left(\mathrm{Mg}_{1 / 3} \mathrm{Nb}_{2 / 3}\right)_{0.45} \mathrm{Ti}_{0.55}\right] \mathrm{O}_{3}$, produces a gaussian peak concentric with the Rayleigh line of the excitation laser used in Brillouin scattering experiments. Such an effect has been used to probe the appearance of the polar nanoregions (PNRs) that emerge above the Curie temperature in these compounds when the center of inversion appears.

In order to understand the origin of the low-energy tail, one approach capable of studying its behavior along the ferroelectric to paraelectric phase transition is needed. The reduction of the off-centering of the $\mathrm{Nb}$ atoms should have a different effect on the low-energy tail depending on its origin. The phase transition can be induced by temperature, but at high temperature, the thermal fluctuations are expected to have a strong contribution in the low energy tail as observed before. ${ }^{7}$ These fluctuations might mask the tail that we intend to study. The phase transition can also be induced under high pressure since in a displacive phase transition as the one undergone by $A \mathrm{BN} x$ relaxor ferroelectrics, the Curie temperature is known to decrease under compression reaching ambient temperature at relatively low pressures. In particular, we have found with high-pressure XRD that CBN28 undergoes the ferroelectric to paraelectric phase transition at ambient temperature and $P_{c}=4 \mathrm{GPa}$, in good agreement with second harmonic generation and Raman spectroscopy studies. ${ }^{12}$

The absorption edge of CBN28 is shown at different pressures in Fig. 2. Under compression, the absorption edge of CBN28 shows almost no shift with pressure with a slight drop at $\sim 4.3 \mathrm{GPa}$ to continue up to the maximum pressure reached $(9.3 \mathrm{GPa})$ almost constant. According to previous studies, ${ }^{12}$

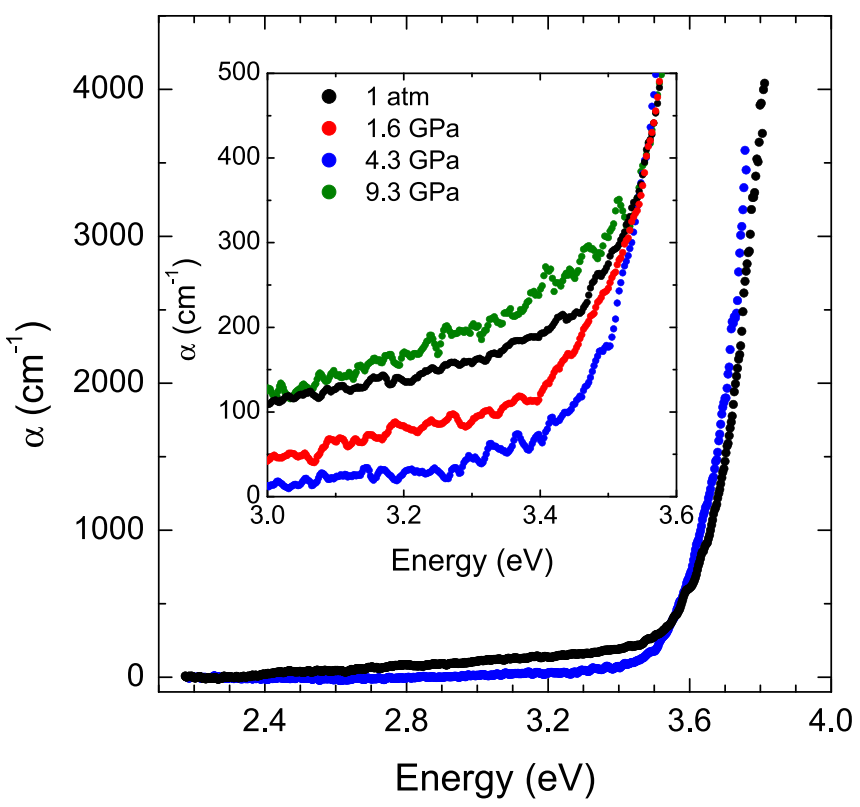

FIG. 2. Absorption spectra of CBN28 at different pressures. The inset shows a zoom of the low-energy tail not accounted for by Urbach's law at different pressures.

static PNRs emerge at $4 \mathrm{GPa}$ in $\mathrm{CBN} 28$ when $\mathrm{Nb}^{5+}$ have almost completely reduced their up-shift along $c$. From $4 \mathrm{GPa}$ to the characteristic pressure $P^{*}=8.5 \mathrm{GPa}$, the $\mathrm{NbO}_{6}^{8-}$ octahedra stop compressing and start tilting to compensate the effect of pressure. Above $P^{*}=8.5 \mathrm{GPa}$, the PNRs become dynamic to finally vanish at the Burns pressure $P_{B}=11.5 \mathrm{GPa}$. Hence, the drop of the absorption edge at $4.3 \mathrm{GPa}$, result of a bandgap decrease of $0.01 \mathrm{eV}$ according to the fit with Urbach's law, is concomitant with the quench of the off-centering of at least one $\mathrm{Nb}^{5+}$ ion supporting our hypothesis about the influence of the $\mathrm{NbO}_{6}^{8-}$ distortion on the bandgap difference between SBN $x$ and CBN28 presented above. No change in the steepness of the absorption spectra is observed under pressure, reflected by a constant $E_{U}=0.112(2) \mathrm{eV}$ up to $9.3 \mathrm{GPa}$. On the other hand, the variation of the low-energy tail of the spectrum can be clearly observed in the inset of Fig. 2. When we start compressing CBN28, the absorption coefficient at $3.3 \mathrm{eV}$ starts decreasing fast up to $4.3 \mathrm{GPa}$ when it has almost vanished and gets masked by the background. However, at higher pressures, it starts to increase reaching values above ambient temperature. This is quantified in Fig. 3 where $\alpha$ at $3.3 \mathrm{eV}$ is plotted as a function of pressure.

This behavior indicates that the origin of this lowenergy tail is most probably the result of the interaction of the pre-edge photons with the ferroelectric domains present in the unpoled CBN28 single crystal used in this experiment. Similar to the origin of the central peak observed by Brillouin scattering ${ }^{27}$ which shows a maximum intensity at $T_{C}$, the light scattering intensity in the pre-edge must be related to the size of the polar domains. Initially, in a normal ferroelectric, polar domains are expected to be large ${ }^{30}$ and reduce their size with pressure until vanishing in the paraelectric phase. However, in a relaxor ferroelectric as CBN28, this process is slowed down and before the spontaneous polarization completely vanishes, nano-sized PNRs appear and remain until $P_{B}$ is reached. ${ }^{12}$ If we assume that the 


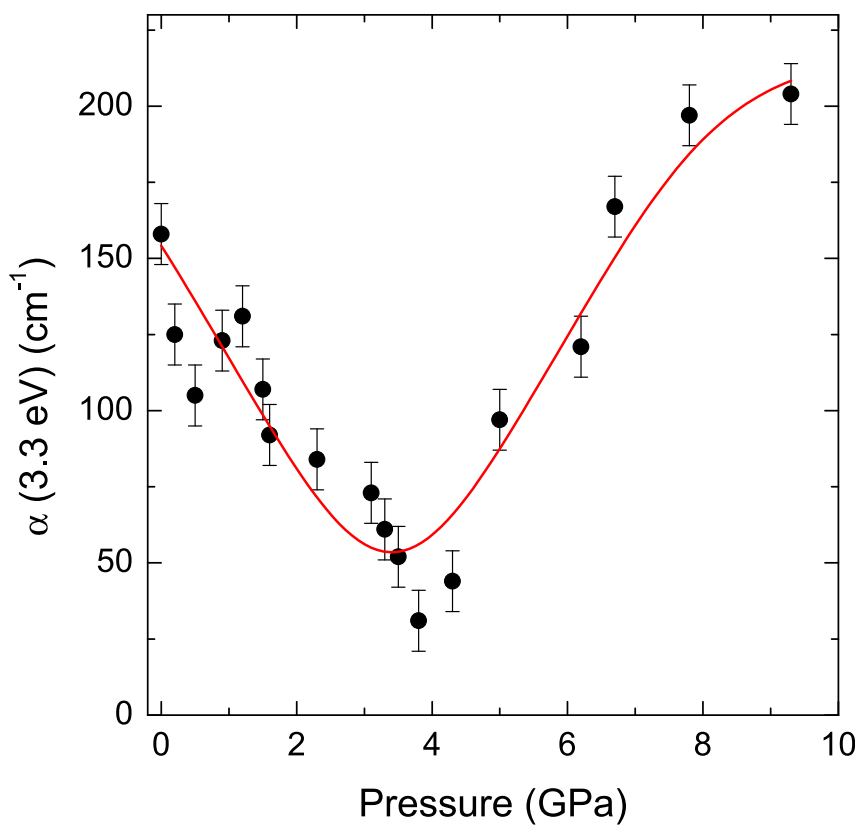

FIG. 3. Pressure dependence of the absorption coefficient $\alpha$ at $3.3 \mathrm{eV}$ for CBN28. The continuous red line is a guide to the eye.

maximum scattering power occurs when the size of the nanodomains is in the order of the scattered photon wavelength in $\mathrm{CBN} 28$, this would take place at $376 \mathrm{~nm}(3.3 \mathrm{eV})$ according to Fig. 2. The larger the domains, the smaller the scattering power, and also, the higher the spontaneous polarization, the higher the scattering power. In this frame, the ferroelectric domains at ambient pressure in the $\mathrm{SBN} x$ family would be around $376 \mathrm{~nm}$ in size, in good agreement with previous piezoforce microscopy works. ${ }^{30}$ Otherwise, according to Fig. 1 and summarized in Table I where the respective values of $\alpha$ at $3.3 \mathrm{eV}$ are shown at ambient pressure, $\alpha$ at $3.3 \mathrm{eV}$ decreases with $\mathrm{Sr}$ incorporation. Considering that Ba-rich $\mathrm{SBN} x$ is a normal ferroelectric and transforms into a relaxor ferroelectric with $\mathrm{Sr}$ incorporation therefore reducing the domain size, this indicates that in $\mathrm{SBN} x$ the spontaneous polarization decrease with $\mathrm{Sr}$ incorporation dominates the scattering factor. Under pressure, the spontaneous polarization decreases ${ }^{12}$ and according to the results shown in Fig. 3, the polar domains increase their size similar to the process that a normal ferroelectric undergoes when approaches the ferroelectric to paraelectric phase. In fact, in CBN28, we find that the absorption coefficient at $3.3 \mathrm{eV}$ and $4.3 \mathrm{GPa}$ is minimum coinciding with the ferroelectric to paraelectric phase transition, but instead of remaining close to zero like in a normal ferroelectric, it starts to increase again under pressure when the PNRs emerge (Fig. 3). Interestingly, at higher pressures, the value of $\alpha$ continues to increase, indicating that despite the density of PNRs is reduced with respect to the polar domains in the ferroelectric phase, their size and scattering power are comparable to those below $4 \mathrm{GPa}$ and even higher when PNRs become dynamic after crossing the characteristic $^{12}$ pressure $P^{*}=8.5 \mathrm{GPa}$. At $9.3 \mathrm{GPa}$, one can observe that the increase with pressure of the absorption coefficient of the pre-edge seems to slow down despite only the last measured point indicates this. Such a tendency change might be correlated with the expected quench of the
PNRs at $11.5 \mathrm{GPa}^{12}$ Unfortunately, the optical absorption experiments were terminated at $9.3 \mathrm{GPa}$ to guarantee hydrostaticity of the pressure medium used and avoid any deterioration of the sample that could have had a direct influence in the absorption spectrum. ${ }^{31}$

Our results show that the fundamental edge of $A_{x} \mathrm{Ba}_{1-x}$ $\mathrm{Nb}_{2} \mathrm{O}_{6}$ tungsten-bronze-type relaxor ferroelectrics has a direct character modeled by an Urbach's tail and takes a value of 3.44(1) $\mathrm{eV}$ for $A=\mathrm{Sr}$ and 3.57(1) $\mathrm{eV}$ for $A=\mathrm{Ca}$. This work demonstrates that the existence of PNRs can be also probed by optical absorption spectroscopy under high pressure providing insight of their interaction with light.

See supplementary material for High-pressure powder $\mathrm{x}$-ray diffraction data and analysis.

J.R.-F. acknowledges the Spanish MINECO for the Juan de la Cierva (IJCI-2014-20513) Program and Dr. Bayarjargal from the Goethe-Universität Frankfurt for providing the CBN28 samples. This work was supported by Spanish MINECO under Grant No. MAT2016-75586-C4-1-P/2-P. The high pressure $\mathrm{X}$-ray diffraction experiments were performed at MSPD beamline at ALBA Synchrotron (Project 2016021588) with the collaboration of ALBA staff.

${ }^{1}$ H. Bach and J. Liebertz, Fortschr. Mineral. 55, 59 (1977).

${ }^{2}$ R. R. Neurgaonkar and W. K. Cory, J. Opt. Soc. Am. (B) 3, 274 (1986).

${ }^{3}$ T. Granzow, T. Woike, M. Wöhlecke, M. Imlau, and W. Kleemann, Phys. Rev. Lett. 89, 127601 (2002).

${ }^{4}$ P. Molina, S. Álvare-García, M. O. Ramírez, J. García-Solé, L. E. Bausá, H. Zhang, W. Gao, J. Wang, and M. Jiang, Appl. Phys. Lett. 94, 071111 (2009).

${ }^{5}$ M. Meyer, M. Wöhlecke, and O. F. Schirmer, Phys. Status Solidi B 221, R1 (2000).

${ }^{6}$ M. Esser, M. Burianek, P. Held, J. Stade, S. Bulut, C. Wickleder, and M. Mühlberg, Cryst. Res. Technol. 38, 457 (2003).

${ }^{7}$ U. Heine, K. Betzler, M. Burianek, and M. Mühlberg, Phys. Status Solidi RRL 4, 166 (2010).

${ }^{8}$ J. Pellicer-Porres, A. Segura, S. Gilliland, A. Muñoz, P. RodríguezHernández, D. Kim, M. S. Lee, and T. Y. Kim, Appl. Phys. Lett. 88, 181904 (2006).

${ }^{9}$ P. B. Jamieson, S. C. Abrahams, and J. L. Bernstein, J. Chem. Phys. 48, 5048 (1968).

${ }^{10}$ H. A. Graetsch, J. Schreuer, M. Burianek, and M. Mühlberg, J. Solid State Chem. 196, 255 (2012).

${ }^{11}$ H. A. Graetsch, C. S. Pandey, J. Schreuer, M. Burianek, and M. Mühlberg, Acta Crystallogr., Sect. B 68, 101 (2012).

${ }^{12}$ J. Ruiz-Fuertes, L. Bayarjargal, B. Winkler, M. Burianek, and M. Mühlberg, Appl. Phys. Lett. 104, 262902 (2014).

${ }^{13}$ P. W. Whipps, Solid State Chem. 4, 281 (1972).

${ }^{14}$ A. Speghini, M. Bettinelli, U. Caldiño, M. O. Ramírez, D. Jaque, L. E. Bausá, and J. García-Solé, J. Phys. D: Appl. Phys. 39, 4930 (2006).

${ }^{15}$ M. Mühlberg, M. Burianek, B. Joschko, D. Klimm, A. Danilewsky, M. Gelissen, L. Bayarjargal, G. P. Görler, and B. Hildmann, J. Cryst. Growth 310, 2288 (2008).

${ }^{16}$ H. K. Mao, P. M. Bell, J. W. Shaner, and D. J. Steinberg, J. Appl. Phys. 49, 3276 (1978).

${ }^{17}$ F. Fauth, I. Peral, C. Popescu, and M. Knapp, Powder Diffr. 28, S360 (2013).

${ }^{18}$ F. Urbach, Phys. Rev. 92, 1324 (1953).

${ }^{19}$ J. Ruiz-Fuertes, A. Friedrich, D. Errandonea, A. Segura, W. Morgenroth, P. Rodríguez-Hernández, A. Muñoz, and Y. Meng, Phys. Rev. B 95, 174105 (2017).

${ }^{20}$ Z. Weibin, W. Weidong, W. Xueming, C. Xinlu, Y. Dawei, S. Changle, P. Liping, W. Yuying, and B. Li, Surf. Interfase Anal. 45, 1206 (2013).

${ }^{21}$ V. Eyert and K.-H. Höck, Phys. Rev. B 57, 12727 (1998). 
${ }^{22}$ R. Lacomba-Perales, D. Errandonea, A. Segura, J. Ruiz-Fuertes, P. Rodríguez-Hernández, S. Radescu, J. López-Solano, A. Mujica, and A. Muñoz, J. Appl. Phys. 110, 043703 (2011).

${ }^{23}$ Y. Zhang, N. A. W. Holzwarth, and R. T. Williams, Phys. Rev. B 57, 12738 (1998).

${ }^{24}$ D. Errandonea, D. Martínez-García, R. Lacomba-Perales, J. Ruiz-Fuertes, and A. Segura, Appl. Phys. Lett. 89, 091913 (2006).

${ }^{25}$ Y. J. Qi, C. J. Lu, J. Zhu, X. B. Chen, H. L. Song, H. J. Zhang, and X. G. Xu, Appl. Phys. Lett. 87, 082904 (2005).

${ }^{26} \mathrm{~B}$. Gu and H. T. Wang, Ferroelectrics-Physical effects (InTech Europe, 2011).
${ }^{27}$ J.-H. Ko and S. Kojima, Appl. Phys. Lett. 91, 082903 (2007).

${ }^{28}$ V. K. Malinovsky, A. M. Pugachev, and N. V. Surovtsev, Bull. Russ. Acad. Sci.: Phys. 74, 1231 (2010).

${ }^{29}$ J.-H. Ko, S. Kojima, A. A. Bokov, and Z.-G. Ye, Appl. Phys. Lett. 91, 252909 (2007).

${ }^{30}$ V. V. Shvartsman, W. Kleemann, T. Lukasiewicz, and J. Dec, Phys. Rev. B 77, 054105 (2008).

${ }^{31}$ J. Ruiz-Fuertes, S. López-Moreno, J. López-Solano, D. Errandonea, A. Segura, R. Lacomba-Perales, A. Muñoz, S. Radescu, P. RodríguezHernández, M. Gospodinov et al., Phys. Rev. B 86, 125202 (2012). 